\title{
Inhibition of BRD4 suppresses the malignancy of breast cancer cells via regulation of Snail
}

\author{
Linlin $\mathrm{Lu}^{1} \cdot$ Zhuojia Chen ${ }^{2} \cdot$ Xinyao $\mathrm{Lin}^{1} \cdot \operatorname{Lin} \mathrm{Tian}^{3} \cdot \mathrm{Qiao} \mathrm{Su}^{4} \cdot \operatorname{Panpan} \mathrm{An}^{1} \cdot$ Wuguo $\mathrm{Li}^{4} \cdot$ Yingmin $\mathrm{Wu}^{1} \cdot \mathrm{Jun}^{\mathrm{Du}}{ }^{1}$. \\ Hong Shan ${ }^{5} \cdot$ Cheng-Ming Chiang ${ }^{6} \cdot$ Hongsheng Wang $\mathbb{D}^{1}$
}

Received: 30 November 2018 / Revised: 19 April 2019 / Accepted: 6 May 2019 / Published online: 21 May 2019

๑) ADMC Associazione Differenziamento e Morte Cellulare 2019

\begin{abstract}
The mechanistic action of bromodomain-containing protein 4 (BRD4) in cancer motility, including epithelial-mesenchymal transition (EMT), remains largely undefined. We found that targeted inhibition of BRD4 reduces migration, invasion, in vivo growth of patient-derived xenograft (PDX), and lung colonization of breast cancer (BC) cells. Inhibition of BRD4 rapidly decreases the expression of Snail, a powerful EMT transcription factor (EMT-TF), via diminishing its protein stability and transcription. Protein kinase D1 (PRKD1) is responsible for BRD4-regulated Snail protein stability by triggering phosphorylation at Ser11 of Snail and then inducing proteasome-mediated degradation. BRD4 inhibition also suppresses the expression of Gli1, a key transductor of Hedgehog $(\mathrm{Hh})$ required to activate the transcription of SNAI1, in BC cells. The GACCACC sequence $(-341$ to -333$)$ in the SNAI1 promoter is responsible for Gli1-induced transcription of SNAI1. Clinically, BRD4 and Snail levels are increased in lung-metastasized, estrogen receptor-negative (ER-), and progesterone receptor-negative (PR-) breast cancers and correlate with the expression of mesenchymal markers. Collectively, BRD4 can regulate malignancy of breast cancer cells via both transcriptional and post-translational regulation of Snail.
\end{abstract}

\section{Introduction}

Bromodomain-containing protein 4 (BRD4) is a member of the BET (Bromodomain and Extra-Terminal) family proteins [1]. It interacts primarily with acetylated histone $\mathrm{H} 4$ at

These authors contributed equally: Linlin Lu, Zhuojia Chen.

Edited by B Dynlacht

Supplementary information The online version of this article (https:// doi.org/10.1038/s41418-019-0353-2) contains supplementary material, which is available to authorized users.

Cheng-Ming Chiang

cheng-ming.chiang@utsouthwestern.edu

$\triangle$ Hongsheng Wang

whongsh@mail.sysu.edu.cn

1 Guangdong Provincial Key Laboratory of New Drug Design and Evaluation, School of Pharmaceutical Sciences, Sun Yat-sen University, Guangzhou, Guangdong 510006, China

2 Sun Yat-sen University Cancer Center, State Key Laboratory of Oncology in South China, Collaborative Innovation Center for Cancer Medicine, Guangzhou, Guangdong 510060, China lysine $(\mathrm{K})$ residues 5, 8, 12 and/or 16 and acetylates histone $\mathrm{H} 3$ at $\mathrm{K} 9$ and/or K14, via its tandem bromodomains, and recruits the transcription machinery to regulate gene expression [2, 3]. BRD4 can also occupy distal enhancer RNA (eRNA) regions to regulate transcription initiation and elongation [4]. Since BRD4 is a potential epigenetic therapeutic target, inhibitors of BET are currently in different phases of preclinical and clinical trials for treatment of multiple tumors [5]. Targeted inhibition of BRD4 can suppress growth and induce apoptosis of various cancer cells, including acute myeloid leukemia [6], diffuse large B

3 Department of Pharmacy, The Fifth Affiliated Hospital of Sun Yat-sen University, Zhuhai, Guangdong 519000, China

4 Laboratory Animal Center, The First Affiliated Hospital of Sun Yat-Sen University, Guangzhou, Guangdong 510080, China

5 Key Laboratory of Biomedical Imaging of Guangdong Province, Guangdong Provincial Engineering Research Center of Molecular Imaging, The Fifth Affiliated Hospital of Sun Yat-sen University, Zhuhai, Guangdong 519000, China

6 Simmons Comprehensive Cancer Center, Department of Pharmacology, and Department of Biochemistry, University of Texas Southwestern Medical Center, Dallas, TX 75390, USA 
cell lymphoma [7], prostate cancer [8], and breast cancer [9].

The roles of BRD4 in cancer metastases are largely unknown. Recent studies revealed that BRD4 can regulate cell motility via regulating Jagged1/Notch1 signaling [10] and interacting with epithelial-mesenchymal transition (EMT) transcription factor (EMT-TF) Twist [11]. EMT is a process by which epithelial cells gain a migratory and invasive mesenchymal phenotype [12]. EMT-TFs, including Twist, Snail, and Zeb1, can trigger the progression of EMT and promote cancer metastasis [13]. Expression of Snail (encoded by SNAI1), a key transcriptional repressor of E-cadherin expression in EMT, is epigenetically regulated by chromatin modifiers such as lysine-specific demethylase1 (LSD1) [14] and Suv39H1 histone methyltransferases [15]. Our recent study revealed that targeting CDK7 increases the stability of Snail to promote colorectal cancer dissemination [16]. The second bromodomain of BRD4 can intact with diacetylated EMT-TF Twist and then activate downstream genes [11]. Considering that BRD4 function is highly context-dependent, we are interested in determining the roles of BRD4 and its regulatory effects on EMT-TFs and dissemination of breast cancer (BC) cells.

JQ1, a small molecule inhibitor of BRD4 [17], has been widely used to investigate the roles of BET proteins [9, 18-20]. In the present study, we evaluated the potential roles of targeted inhibition of BRD4 by the use of JQ1 and its specific siRNA. We found BRD4 was involved in in vitro and in vivo dissemination of $\mathrm{BC}$ cells. Targeted inhibition of BRD4 also suppressed EMT progression via protein kinase D1 (PRKD1)-mediated post-translational and Gli1-mediated transcriptional downregulation of Snail.

\section{Results}

\section{BRD4 is upregulated in BC and modulates the malignancy of cancer cells}

We first measured the expression of BRD4 in various cells. Both mRNA (Fig. S1A) and protein (Fig. 1a) levels of BRD4 in $\mathrm{BC}$ cell lines were greater than that of nontumorigenic BC cell lines (HBL100 and MCF-10A). Consistently, increased expression of BRD4 in BC versus normal tissue has been observed in TCGA, Crutis, and Finak data from Oncomine databases (Fig. 1b), suggesting BRD4 is generally upregulated in BC cells and tissues.

We then evaluated the potential roles of BRD4 using BET bromodomian inhibitor JQ1. JQ1 $>5 \mu \mathrm{M}$ significantly decreased the proliferation of BC cells (Fig. S1B). Further, JQ1 $(1 \mu \mathrm{M}$, which had no effect on cell proliferation, Fig. S1B) significantly inhibited the migration of MDA-
MB-231 cells, even in the presence of TGF- $\beta$, which could induce EMT of cancer cells (Fig. 1c). Similarly, JQ1 inhibited the migration of BT-549 cells in the absence or presence of TGF- $\beta$ (Fig. S1C). Transwell assay confirmed that JQ1 could inhibit the invasion of BC cells (Fig. 1d). To avoid the off-target effect of JQ1, we knocked down the expression of BRD4 using two specific siRNAs (Fig. S1D). si-BRD4 significantly inhibited the migration of BC cells (Fig. 1e). However, knockdown of BRD2 and BRD3 (Fig. S1E), which are also inhibited by JQ1, had no effect on cell migration (Fig. S1F). Conversely, overexpression of BRD4 (Fig. S1G) triggered wound closure of BC cells (Fig. 1f).

The mesenchymal marker fibronectin (FN) was checked in JQ1-treated BC cells. JQ1 inhibited the expression of FN and further blocked TGF- $\beta$-induced $\mathrm{FN}$ in BC cells (Fig. 1g). Moreover, JQ1 suppressed the expression of FN in a concentration- and time-dependent manner (Fig. 1h). Similarly, knockdown of BRD4 downregulated FN expression in BC cells (Fig. 1i), while overexpression of BRD4 increased the expression of FN (Fig. S1G). To test the in vivo effects of JQ1, we established high lung metastasis potential BC cell models [21] as summarized in Fig. S2 and named them as MDA-MB-231 ${ }^{\text {LMF3 }}$ or BT-549 ${ }^{\text {LMF3 }}$ cells. JQ1 could suppress the expression of FN in high lung metastasis potential $\mathrm{BC}$ cells also in a dose-dependent manner (Fig. S1H). Collectively, BRD4 is upregulated in $\mathrm{BC}$ and modulates the malignancy of $\mathrm{BC}$ cells.

\section{JQ1 suppresses the in vivo growth of PDX tumors and lung colonization of BC cells}

To further examine the in vivo effects of JQ1, mice implanted with a TNBC patient-derived xenograft (PDX) model were treated with vehicle or JQ1. At the end of the experiment, tumor size, volume, and weight in the JQ1injected group were significantly lower than those measured in the control group (Fig. 2a-d). No significant change of body weight was observed between control and JQ1 groups (data not shown). Additionally, Ki67-positive staining, which recognizes a nuclear antigen expressed in proliferating cells, in the JQ1 group was decreased in vivo (Fig. 2e). This result suggested that JQ1 could inhibit in vivo growth of PDX BC.

IHC data showed that JQ1 decreased the expression of FN in the PDX model (Fig. 2e). To further determine the impact of JQ1 on in vivo progression, MDA-MB-231 $1^{\text {LMF3 }}$ cells were injected into the lateral tail vein to get lung colonization. As shown in Fig. 2f, g, the number and size of lung tumors derived from JQ1-treated MDA-MB-231 $1^{\mathrm{LMF} 3}$ cells were significantly decreased compared to that of untreated cells. Collectively, targeted inhibition of BRD4 decreased in vivo progression of $\mathrm{BC}$. 
A

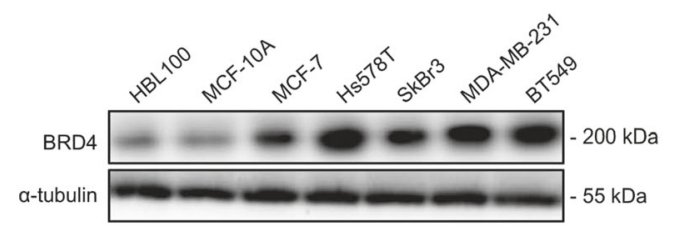

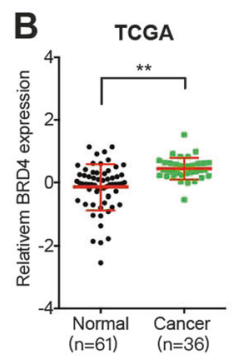
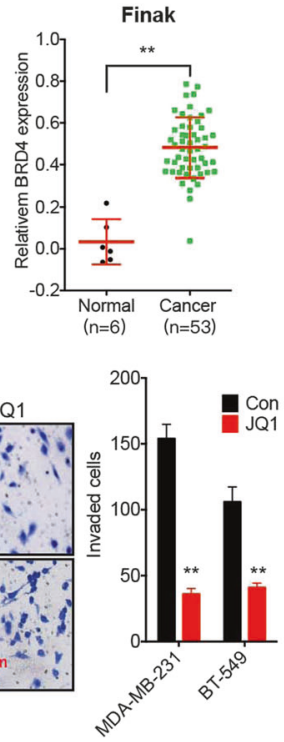

Con
C
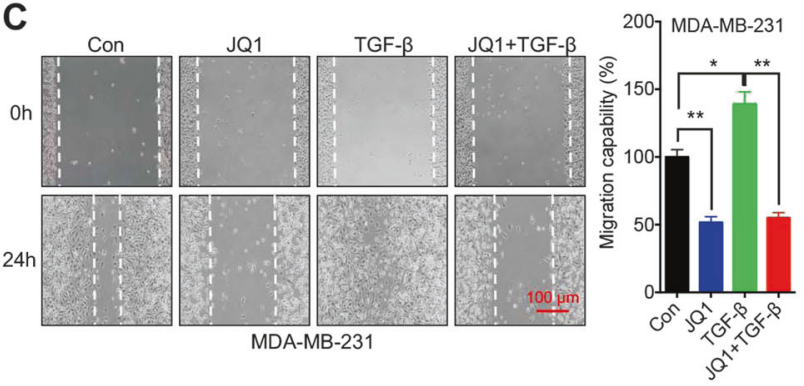

E
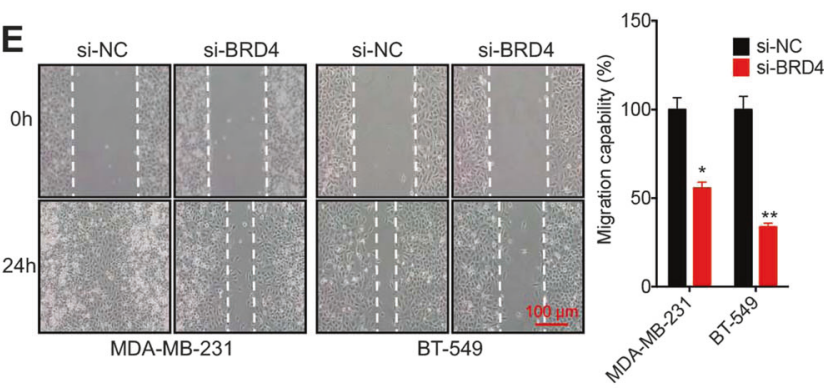

$\mathbf{F}$
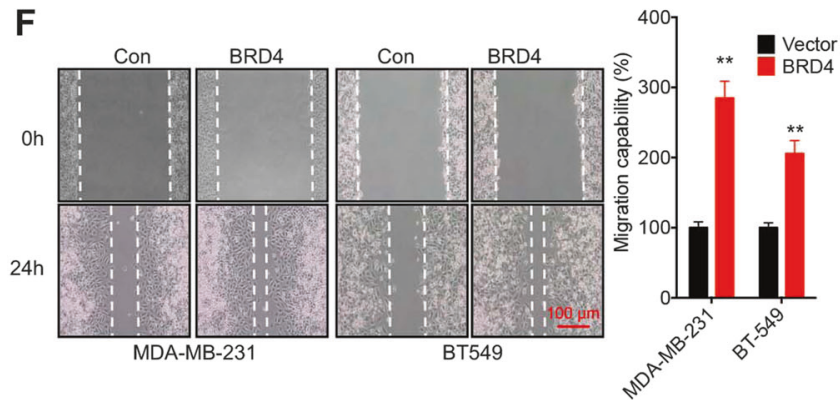

D
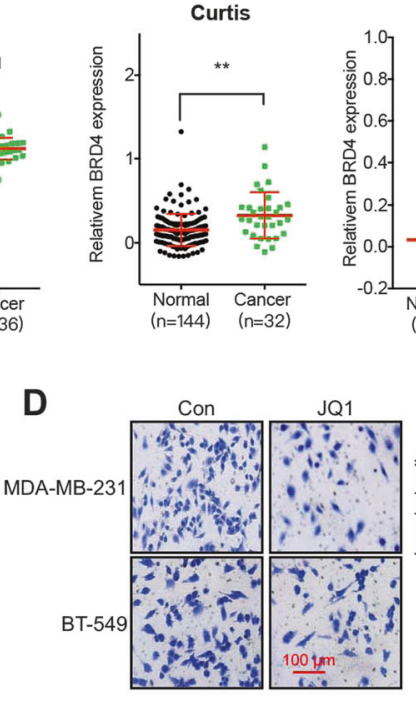
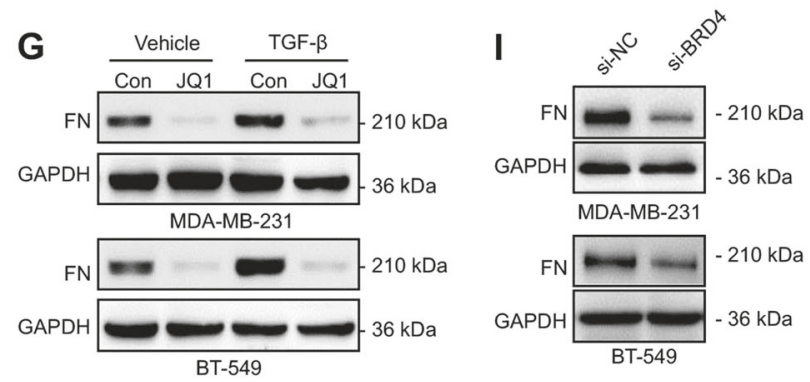

H

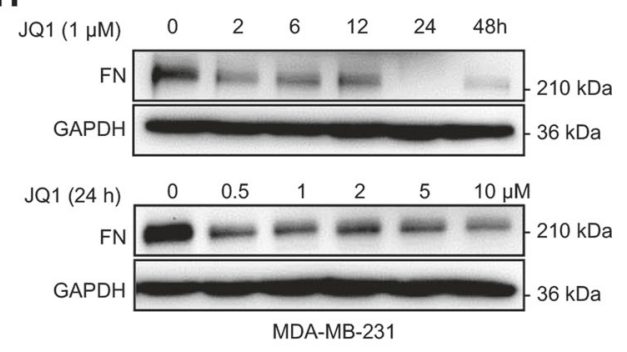

Fig. 1 BRD4 is upregulated in breast cancers and modulates the malignancy of cancer cells. a Protein expression of BRD4 in breast cancer cells and non-tumorigenic breast cancer cell lines (HBL100 and MCF-10A); b Relative mRNA expression of BRD4 in breast and adjacent normal tissue based on data available from the TCGA database and Oncomine database with Curtis and Finak cancer, respectively; c MDA-MB-231 cells were treated with vehicle, JQ1 $(1 \mu \mathrm{M})$, TGF- $\beta(10 \mathrm{ng} / \mathrm{ml})$, alone or together, for $24 \mathrm{~h}$. Wound healing was recorded (left) and quantitatively analyzed (right); d BC cells were treated with vehicle or JQ1 $(1 \mu \mathrm{M})$ for $24 \mathrm{~h}$, followed by in vitro invasion checked by transwell chamber (left) and quantitatively analyzed (right); e BC cells were transfected with

\section{Snail is involved in BRD4-regulated malignancy of BC cells}

We performed mRNA-seq in MDA-MB-231 cells treated with or without JQ1 for $24 \mathrm{~h}$. Gene Set Enrichment
si-NC or si-BRD4 for $24 \mathrm{~h}$. Wound healing was recorded (left) and quantitatively analyzed (right); f BC cells were transfected with vector control or a BRD4 construct for $24 \mathrm{~h}$. Wound healing was recorded (left) and quantitatively analyzed (right); $\mathbf{g}$ BC cells were treated with vehicle, JQ1 $(1 \mu \mathrm{M})$, TGF- $\beta(10 \mathrm{ng} / \mathrm{ml})$, alone or together, for $24 \mathrm{~h}$. FN was checked by western blot analysis; h MDA-MB-231 cells were treated with JQ1 $(1 \mu \mathrm{M})$ for increasing time periods (up) or increasing doses of JQ1 (down) for $24 \mathrm{~h}$. FN was checked by western blot analysis; i After transfection with si-NC or si-BRD4 for $24 \mathrm{~h}, \mathrm{FN}$ in BC cells was checked by western blot analysis. Data were presented as means \pm SD from three independent experiments. $* p<0.05, * * p<0.01$ compared with control

Analysis (GSEA) revealed that the JQ1 treatment gene expression profile was negatively associated with wound healing and EMT of cancer cells (Fig. 3a), suggesting that JQ1 treatment suppressed wound closure and EMT. We then checked the expression of EMT-related transcription 
Fig. 2 JQ1 suppresses in vivo growth of PDX tumors and lung colonization of BC cells. Tumor growth curves of patient-derived tumors treated with or without JQ1 at indicated time intervals; b-d Tumor images (b), volumes (c), and weights (d) of patientderived tumors treated with or without JQ1 at the end of the experiment; e IHC (Ki-67 and fibronectin)-stained paraffinembedded sections obtained from xenografts (left) and quantitatively analyzed (right); f, $g$ MDA-MB-231 $1^{\mathrm{LMF} 3}$ cells were injected into nude mice via tail vein injection.

Representative images of metastatic lung tumors and the H\&E staining results were shown (f), with the number of lung tumors derived from MDA-MB-231 $1^{\text {LMF3 }}$ cells in control and JQ1 groups quantitatively analyzed (g). Data were presented as means \pm SD from three independent experiments. $* * p<0.01$ compared with control

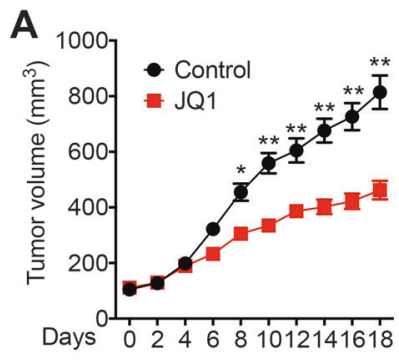

B
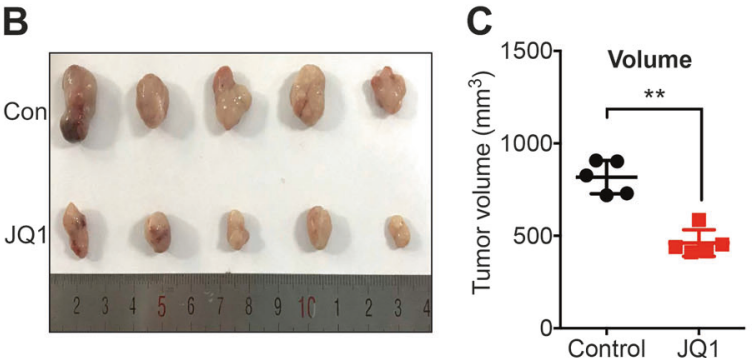

D

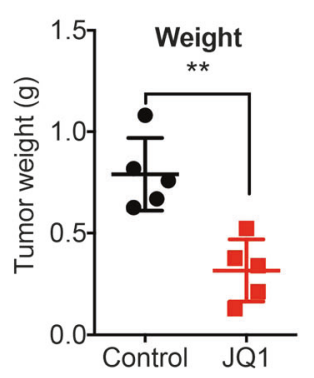

E
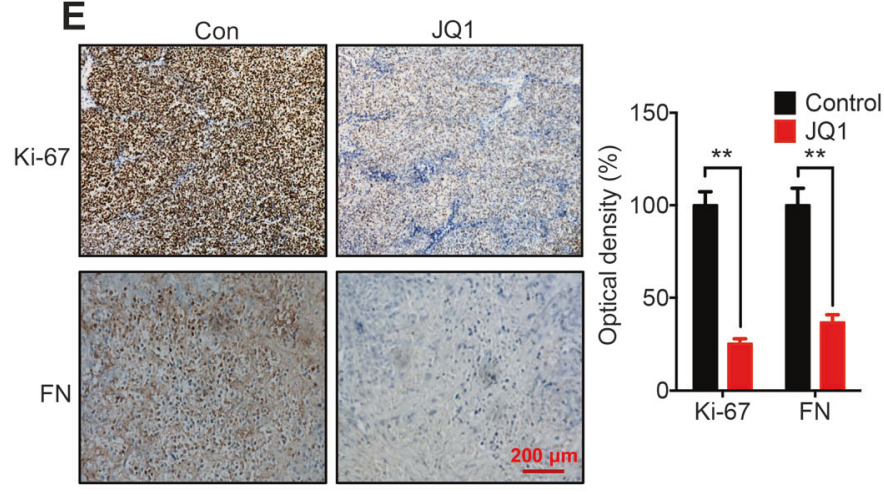

$\mathbf{F}$

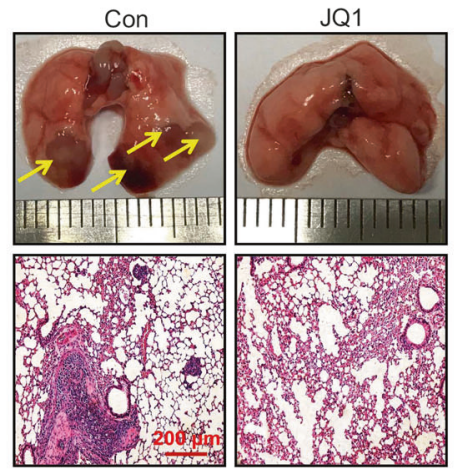

G

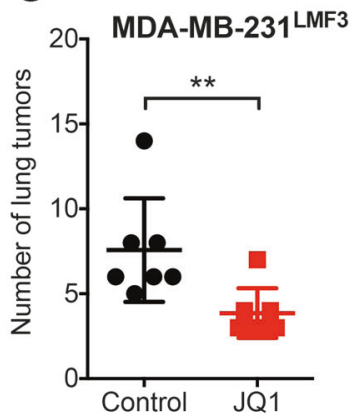

factors (EMT-TFs) including Snail, Slug, Twist, and Zeb1 in BC cells treated with JQ1. JQ1 decreased the expression of Snail, but not Slug, Twist and Zeb1, in both MDA-MB231 (Fig. 3b) and BT-549 (Fig. S3A) cells. Further, JQ1 could decrease the expression of Snail in 2-h treatment in both MDA-MB-231 (Fig. 3c) and BT-549 (Fig. S3B) cells. The JQ1-induced rapid decrease of Snail could not be reversed even in the present of TGF- $\beta$ in BC cells (Fig. 3d). Confocal imaging revealed that expression and nuclear accumulation of Snail was markedly decreased in JQ1treated MDA-MB-231 (Fig. 3e). Subcellular fractionation and western blot analysis confirmed that JQ1 significantly inhibited nuclear accumulation of Snail in BT-549 cells (Fig. S3C). Consistently, si-BRD4 markedly inhibited the expression of Snail in BC cells (Fig. 3f), while overexpression of BRD4 increased the expression and nuclear accumulation of Snail (Fig. 3g). Neither si-BRD2 nor siBRD3 altered the expression of Snail in MDA-MB-231 cells (Fig. S3D). In the PDX model, IHC data showed that JQ1 decreased the expression of Snail in xenograft models (Fig. 3h).

Since Snail is known to be a potent EMT-TF able to trigger cancer progression [22], we verified its role in JQ1suppressed migration of BC cells. The inhibitory effect of JQ1 on the migration of MDA-MB-231 cells were $46 \%$ and $71 \%$, respectively, in cells without or with ectopic Snail expression (Fig. 3i), suggesting that overexpression of Snail could attenuate JQ1-inhibited cell migration. Overexpression of Snail also attenuated JQ1-suppressed FN in MDA-MB-231 (Fig. 3j) and BT-549 (Fig. S3E) cells. These results indicated that Snail is involved in BRD4-regulated malignancy of BC cells. Further, JQ1 decreased Snail mRNA in both MDA-MB-231 (Fig. 3k) and BT-549 (Fig. S3F) after $12 \mathrm{~h}$ treatment. Collectively, JQ1 could suppress the malignancy of BC cells via decreasing Snail expression. 
A

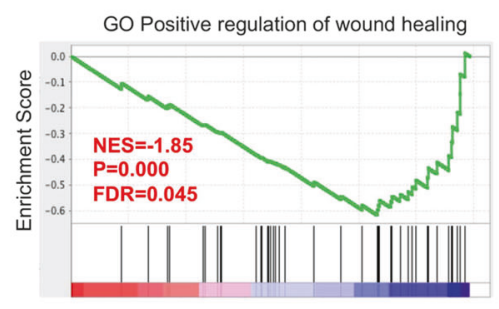

C

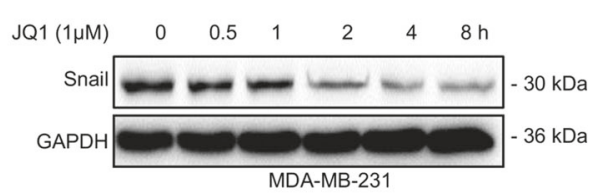

E
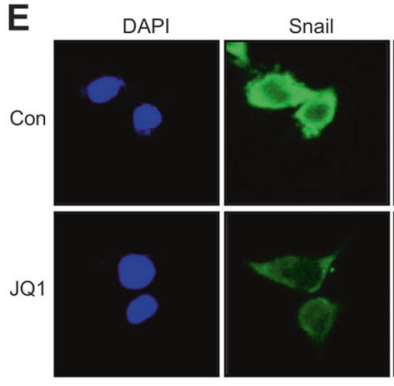

MDA-MB-231

I

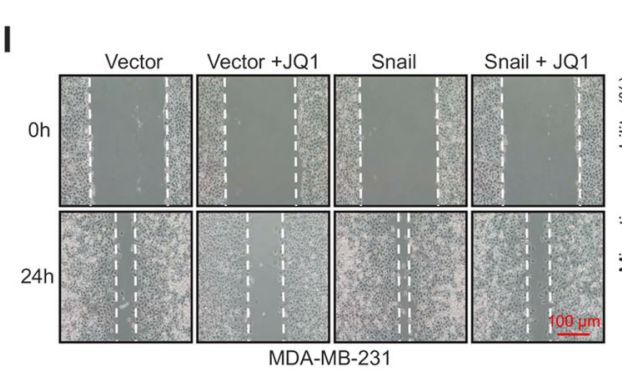

MDA-MB-231

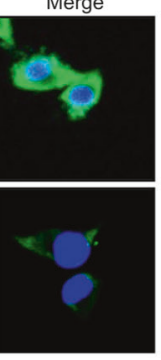

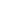

Epithelial mesenchymal transition

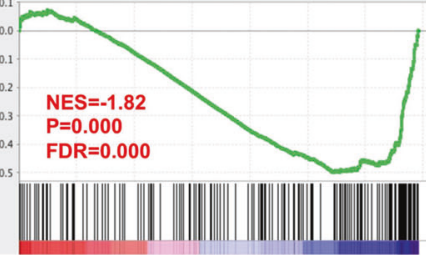

B

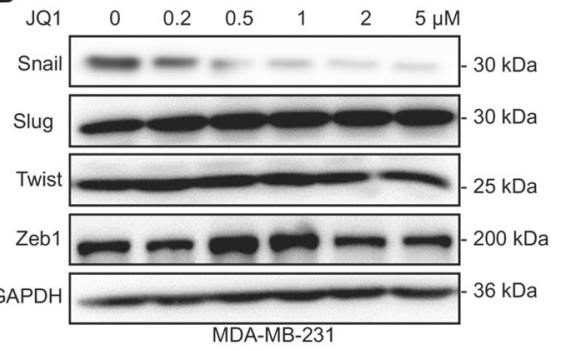

D

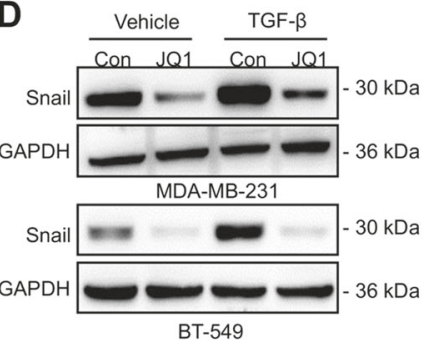

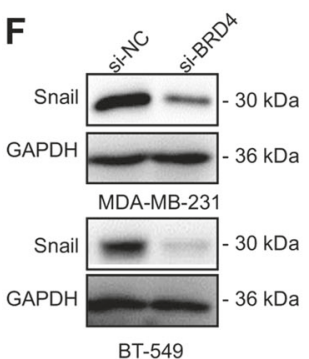

Snail

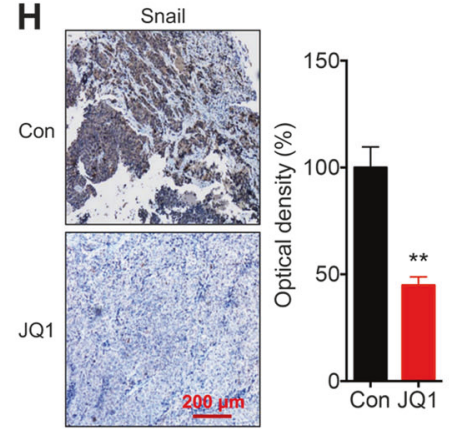

G

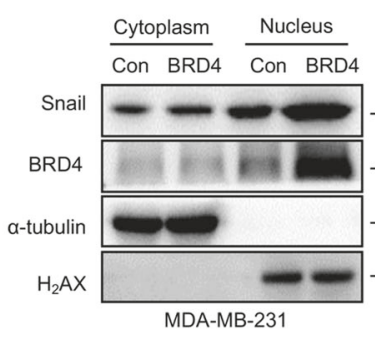

K

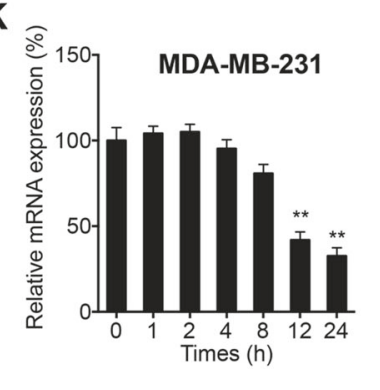

J

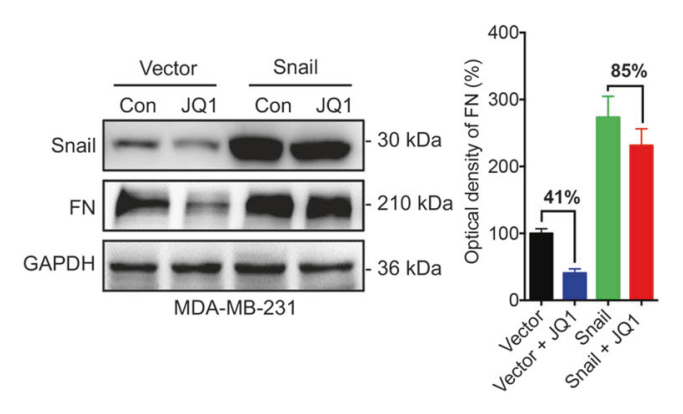


Fig. 3 Snail is involved in BRD4-regulated malignancy of BC cells. a GSEA reveals negative enrichment of JQ1-altered genes in wound healing and EMT gene sets; b MDA-MB-231 cells were treated with increasing concentrations of JQ1 for $24 \mathrm{~h}$. The expression of EMT-TFs was checked; c MDA-MB-231 cells were treated with JQ1 $(1 \mu \mathrm{M})$ for the increasing time periods. The expression of Snail was checked by western blot analysis; $\mathbf{d}$ BC cells were treated with vehicle, JQ1 $(1 \mu \mathrm{M})$, TGF $-\beta(10 \mathrm{ng} / \mathrm{ml})$, alone or together, for $24 \mathrm{~h}$. The expression of Snail was checked by western blot analysis; e MDA-MB-231 cells were treated with or without JQ1 $(1 \mu \mathrm{M})$ for $24 \mathrm{~h}$. The expression of Snail was checked by immunofluorescence staining (green). Nuclei were visualized with DAPI staining (blue); $\mathbf{f}$ After transfection with si$\mathrm{NC}$ or si-BRD4 for $24 \mathrm{~h}$, the expression of Snail in BC cells was checked by western blot analysis; $\mathrm{g}$ MDA-MB-231 cells were transfected with pcDNA (vector) or pcDNA/BRD4 for $24 \mathrm{~h}$. The expression of Snail in subcellular fractions was checked by western blot analysis; $\mathbf{h}$ The expression of Snail in paraffin-embedded sections obtained from xenografts was measured by IHC (left) and quantitatively analyzed (right); I, j MDA-MB-231 cells were transfected with pcDNA (vector) or pcDNA/Snail for $12 \mathrm{~h}$ and then further treated with or without JQ1 $(1 \mu \mathrm{M})$ for $24 \mathrm{~h}$. I Wound healing was recorded (left) and quantitatively analyzed (right), and $\mathbf{J}$ the expression of FN and Snail was checked by western blot analysis (left) and quantitatively analyzed (right); k MDA-MB-231 cells were treated with JQ1 $(1 \mu \mathrm{M})$ for the increasing time periods. The mRNA expression of Snail was checked by $\mathrm{qRT}$-PCR. Data were presented as means \pm SD from three independent experiments. ${ }^{* *} p<0.01$ compared with control

\section{BRD4 post-translationally regulates Snail protein stability via PRKD1 in BC cells}

Our data showed downregulation of Snail protein $(2 \mathrm{~h}$, Fig. 3c) was much more rapidly than its effect on Snail mRNA (12 h, Fig. 3k), suggesting that JQ1 might regulate the stability of Snail protein. Protein stability assay showed that JQ1 decreased the half-life of Snail in both MDA-MB231 (Fig. 4a) and BT-549 (Fig. S4A) cells. In the presence of proteasome-mediated degradation inhibitor MG-132, JQ1-induced downregulation of Snail was significantly attenuated (Fig. 4b). Further, immunoprecipitation results showed that ubiquitylation of Snail was dramatically increased in JQ1-treated MDA-MB-231 and BT-549 cells (Fig. 4c). Interestingly, IP results showed that Snail associated with BRD4, but their interaction was not affected by JQ1 treatment (Fig. 4d). This was further confirmed by quantitative analysis of the pulldown versus input signals (Fig. S4B) and also by reciprocal immunoprecipitation with BRD4 antibody that efficiently pulled down Snail (Fig. S4C). Since Snail could be acetylated by p300 acetyltransferase [23], we further examined whether Snail acetylation might play a role in BRD4 interaction that could be potentially disrupted by JQ1. As shown with total Snail, acetyl-Snail and BRD4 signals in the IP were not diminished by JQ1 in both MDA-MB-231 and BT-549 cells (Fig. 4d and Fig. S4B), suggesting that JQ1 had no effect on either Snail acetylation or BRD4-Snail interaction.
Collectively, these data suggest that JQ1 regulates protein stability and ubiquitylation of Snail and the Snail-BRD4 interaction likely occurs independent of Snail acetylation.

GSK-3 $\beta$ is a major kinase that phosphorylates Snail and induces its degradation [24], while activation of CSN2 blocks the ubiquitination and degradation of Snail [25]. However, JQ1 had no effect on GSK-3 $\beta$ phosphorylation and CSN2 expression (Fig. S4D). Further, JQ1 had no effect on subcellular localization of GSK-3 $\beta$ (Fig. S4E). In addition, the relative binding between GSK-3 $\beta$ and CSN2 with Snail in MDA-MB-231 and BT-549 cells was independent of JQ1 treatment (Fig. 4d). The respective inhibitors of GSK-3 $\beta$, p65, MAPK/ERK, and PI3K/Akt cannot reverse the JQ1-decreased Snail in BC cells (Fig. S4F). These data indicated that JQ1-downregulated Snail occurs regardless of GSK- $3 \beta$ and NF- $\mathrm{kB}$.

To systematically investigate the factors involved in JQ1-induced destabilization of Snail, we analyzed the mRNA expression of 21 factors related to post-translational regulation of Snail stability or nuclear turnover of Snail (Summarized at Table S1). JQ1 had no significant effect on most of the factors including GSK-3 $\beta$ and CSN2, while dramatically increased the expression of PRKD1 (Fig. 4d, e), which can phosphorylate Ser11 of Snail and trigger its degradation via ubiquitylation [26]. JQ1 increased the protein level of PRKD1 in BC cells in 2-h treatment (Fig. 4f). Consistently, si-BRD4 increased PRKD1, while overexpression of BRD4 decreased PRKD1, in MDA-MB-231 cells (Fig. S4G). However, si-BRD2 and si-BRD3 had no effect on PRKD1 expression (Fig. S4H). In PDX tumors, IHC data showed that JQ1 increased the expression of PRKD1 (Fig. 4g). Overexpression of PRKD1 decreased the expression of Snail in BC cells (Fig. S4I), while its inhibitor CID 755763 (CID) increased the half-life of Snail protein in MDA-MB-231 cells (Fig. S4J). To verify PRKD1-induced degradation of Snail via Ser11 phosphorylation, we mutated Snail Ser11 to Ala. The efficiency of JQ1-induced downregulation of Snail in MDA-MB-231 was attenuated by mutation of Snail at Ser11 (Fig. 4h). Mutation of Snail Ser11 increased the half-life of Snail (Fig. S4K). Further, Ser 11 mutation abolished PRKD1 inhibitor (CID)-induced extension of Snail half-life (Fig. S4K). JQ1 significantly increased Ser11 phosphorylation of Snail in BC cells (Fig. S4L). Knockdown of PRKD1 attenuated JQ1-induced downregulation of Snail and FN (Fig. 4i). Overexpression of BRD4 increased the expression of Snail while decreased its phosphorylation at Ser11; however, these effects were abolished in the present of CID (Fig. S4M). These results indicate that BRD4regulated phosphorylation of Snail is PRKD1-dependent. It also confirmed that PRKD1 is involved in BRD4-regulated Snail protein stability and EMT in BC cells. 
A
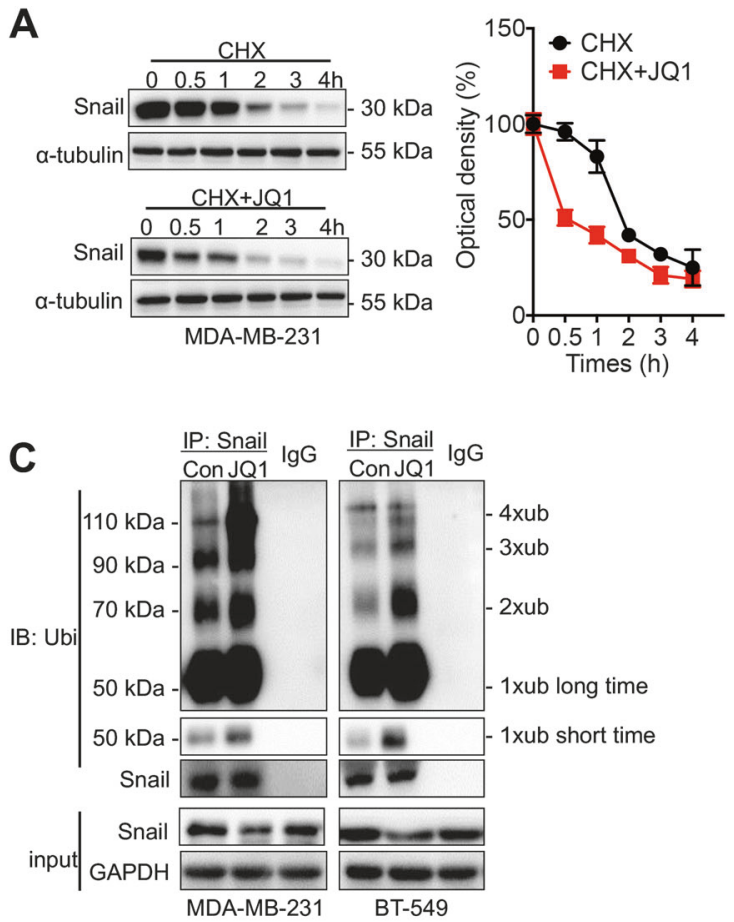

E

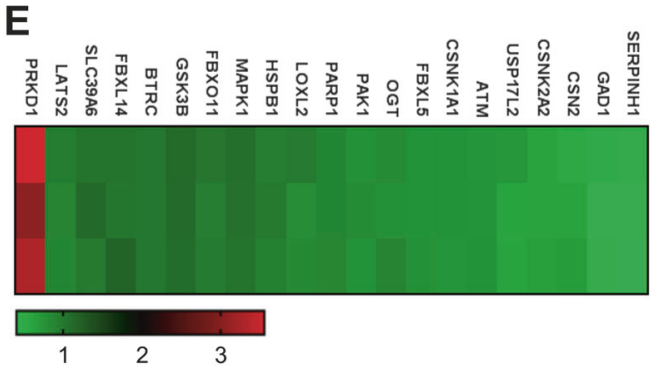

B
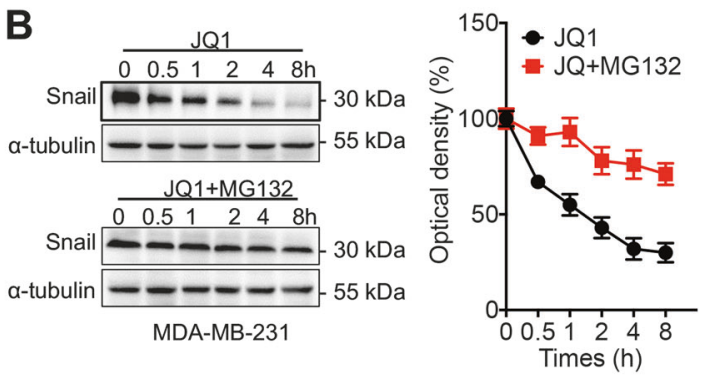

H
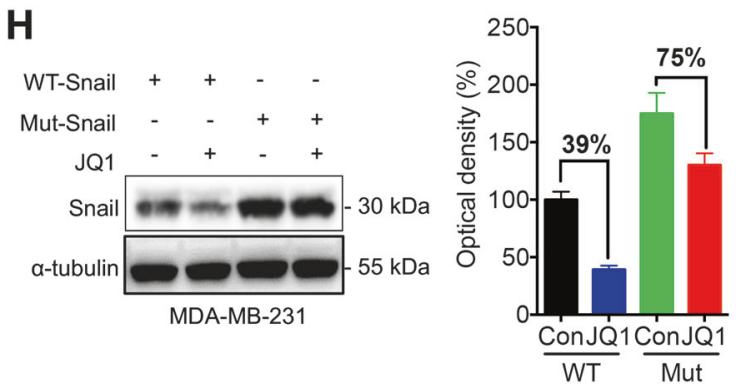

\section{BRD4 transcriptionally regulates Snail expression via Gli1 in BC cells}

We showed that JQ1 suppressed mRNA expression of Snail after treatment for $12 \mathrm{~h}$ (Fig. 3k \& S3F). Consistently, si-BRD4 also decreased the mRNA expression of Snail after transfection for $24 \mathrm{~h}$ (Fig. 5a). However, neither si-BRD2 nor si-BRD3 influenced the mRNA expression of Snail in MDA-MB-231 cells (Fig. S5A).
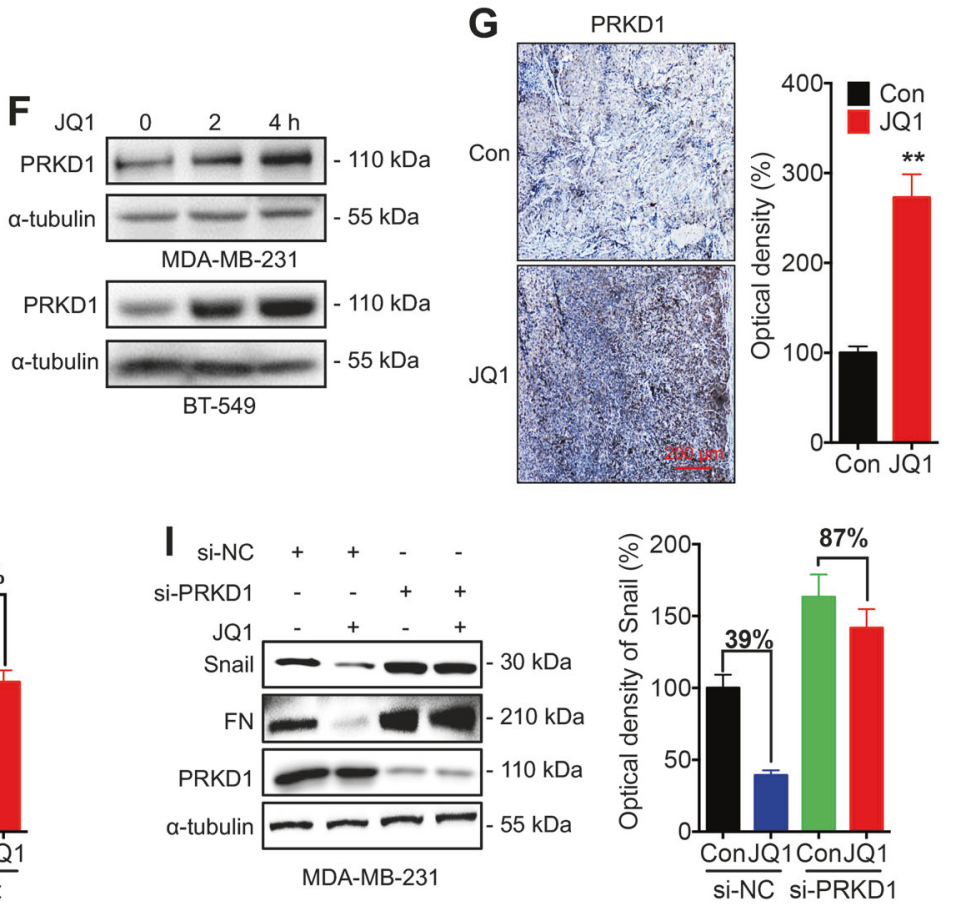

This was PRKD1-independent since si-PRKD1 had no effect on Snail mRNA expression (Fig. S5B). JQ1 did not affect the stability of Snail precursor mRNA (pre-mRNA, Fig. S5C) or mature mRNA (mat-mRNA, Fig. S5D). Further, JQ1 had no effect on the nuclear export of Snail mRNA in MDA-MB-231 cells (Fig. S5E). These results suggested that BRD4-regulated mRNA expression of Snail might not be related to RNA stability or nuclear turnover. 
Fig. 4 BRD4 post-translationally regulates Snail via PRKD1 in BC cells. MDA-MB-231 cells were treated with $10 \mu \mathrm{g} / \mathrm{ml} \mathrm{CHX}$ in the present or absence of JQ1 $(1 \mu \mathrm{M})$ for the indicated time periods. The expression of Snail was detected by western blot analysis (left) and quantitatively analyzed (right); a MDA-MB-231 cells were treated with MG132 $(5 \mu \mathrm{M})$ in the present or absence of JQ1 $(1 \mu \mathrm{M})$ for the indicated time periods. The expression of Snail was detected by western blot analysis (left) and quantitatively analyzed (right); b MDA-MB-231 or BT-549 cells were treated with or without JQ1 $(1 \mu \mathrm{M})$ for $2 \mathrm{~h}$ in the presence of MG-132. After Snail was immunoprecipitated, the ubiquitination of Snail was examined by western blot analysis. An equal amount of Snail was loaded after IP according to a pre-western blot; c MDA-MB-231 or BT-549 cells were treated with or without JQ1 $(1 \mu \mathrm{M})$ for $2 \mathrm{~h}$. After Snail was immunoprecipitated, the binding between Snail and other proteins was examined by western blot analysis. An equal amount of Snail was loaded for normalization according to a pre-western blot; d MDA-MB-231 cells were treated with JQ1 $(1 \mu \mathrm{M})$ for $1 \mathrm{~h}$. The mRNA expression of Snail protein stability-related factors was checked by qRT-PCR; e After treating cells with JQ1 $(1 \mu \mathrm{M})$ for the indicated time periods, the expression of PRKD1 in MDA-MB-231 and BT-549 cells was examined by western blot analysis; $\mathbf{f}$ The expression of PRKD1 in paraffin-embedded sections obtained from xenografts was measured by IHC (left) and quantitatively analyzed (right); $\mathbf{g}$ After transfection with a WT or S11A (Mut) Snail construct for $24 \mathrm{~h}$, MDA-MB-231 cells were further treated with or without JQ1 $(1 \mu \mathrm{M})$ for $2 \mathrm{~h}$. The expression of Snail was detected by western blot analysis (left) and quantitatively analyzed (right); h After transfection with si-NC or si-PRKD1 for $24 \mathrm{~h}$, MDAMB-231 cells were further treated with or without JQ1 $(1 \mu \mathrm{M})$ for $2 \mathrm{~h}$. The expression of Snail and FN was detected by western blot analysis (left). The levels of Snail were quantitatively analyzed (right); Data were presented as means $\pm \mathrm{SD}$ from three independent experiments. $* * p<0.01$ compared with control

We then hypothesized that BRD4 might regulate the transcription of Snail in BC cells. Dual luciferase assay showed that JQ1 treatment for $12 \mathrm{~h}$, but not for $1-4 \mathrm{~h}$, significantly inhibited Snail promoter activity (Fig. 5b). Further, JQ1 decreased the expression of pre-Snail mRNA after 12and 24-h treatments (Fig. 5c), suggesting that JQ1 could suppress the transcription of Snail after treatment for $12 \mathrm{~h}$ in $\mathrm{BC}$ cells.

We then checked the mRNA expression of 11 transcription factors potentially regulating the expression of Snail (Summarized at Table S2). JQ1 treatment significantly decreased the mRNA expression of Gli1, but not others, in MDAMB-231 cells (Fig. 5d). Further, JQ1 treatment decreased the protein expression of Gli1 after treatment for $6 \mathrm{~h}$ in BC cells (Fig. 5e). Knockdown of BRD4 suppressed the mRNA expression (Fig. 5f) and decreased the nuclear accumulation (Fig. 5g) of Gli1, while overexpression of BRD4 increased the expression of Gli1 in BC cells (Fig. S5F). Myf5 [27] and ABCG2 [28] are downstream genes directly regulated by Gli1. Downregulation of Myf5 and ABCG2 expression was observed in cells treated with JQ1 for $8 \mathrm{~h}$ (Fig. S5G). IHC data showed that JQ1 decreased the expression of Gli1 in xenograft PDX models (Fig. 5h). These results suggest that BRD4 regulates the expression, nuclear translocation, and transcriptional activity of Gli1 in BC cells.
We then overexpressed Gli1 in MDA-MB-231 cells via transfection of pcDNA/Gli1, which increased the expression of Snail and FN (Fig. 5i). Further, JQ1-suppressed Snail expression ( $41 \%$ of control) was significantly attenuated in cells transfected with pcDNA/Gli1 $(81 \%$ of control) (Fig. 5i). Consistently, JQ1-suppressed FN (Fig. 5i) and JQ1-suppressed cell migration (Fig. 5j) were both reversed by overexpression of Gli1. These data confirmed that Gli1 is involved in BRD4-regulated Snail expression and migration of $\mathrm{BC}$ cells.

Snail is a direct transcriptional target of Gli1 $[29,30]$, but the binding of Gli1 to the Snail promoter has not been well studied. Gli1, a zinc-finger transcription factor, binds to the conserved sequence, 5'-GACCACCCA-3', in its target promoters [31]. Analysis of the region $2.0 \mathrm{~kb}$ upstream of the transcription start site in the SNAII promoter revealed six putative Gli1-binding sites that exhibit $78 \%$ homology to the consensus Gli1-binding sequence (Fig. 5k). ChIPqPCR showed that binding of Gli1 to the potential binding site " $e$ " was much greater than to the other potential sites in MDA-MB-231 cells (Fig. 51). We mutated bind site " $e$ " (to CAGGAGCGG) to generate pGL3-Snail-eMut-Luc and " $f$ " (to CAGGAGCTG) to generate pGL3-Snail-fMut-Luc, respectively (Fig. 5m). JQ1 significantly inhibited the promoter activity of pGL3-Snail-WT-Luc and pGL3-SnailfMut-Luc, while mutation of site "e" significantly decreased Snail promoter activity as well as its sensitivity to JQ1 treatment (Fig. 5n). These results suggest that BRD4upregulated Gli1 directly activates the transcription of Snail via binding to its promoter-proximal site.

\section{BRD4/Snail axis in the progression of BC}

Increased expression of FN, Snail, and BRD4 was observed in MDA-MB-231 ${ }^{\mathrm{LMF} 3}$ and BT-549 ${ }^{\mathrm{LMF} 3}$ cells compared to their parental cells (Fig. 6a), indicating an involvement of BRD4/Snail in the progression of cancer metastasis. Further, both qRT-PCR (Fig. S6A) and IHC (Fig. 6b) showed enhanced expression of Snail, BRD4, and FN in metastasized lung tumors than that in the primary tumors isolated from MMTV-PyMT mice, confirming that BRD4/Snail axis triggers the progression of $\mathrm{BC}$.

Data from LinkedOmics [32] showed expression of BRD4 positively correlated $(p<0.05)$ with the expression of FN1 (Fig. 6c), MMP9 (Fig. S6B), and CDH2 (Fig. S6C) in $880 \mathrm{BC}$ patients, suggesting that BRD4 might be able to trigger EMT progression in the human body. Significant positive correlation was also observed between BRD4 and SNAI1 (Fig. 6d) and between SNAI1 and Gli1 (Fig. 6e) in 880 BC patients. We then analyzed the expression of BRD4/Snail axis and their correlation with clinical characteristics of BC. The expression of BRD4 in basal BC was significantly $(p<0.01)$ greater than that of other subtypes 
A

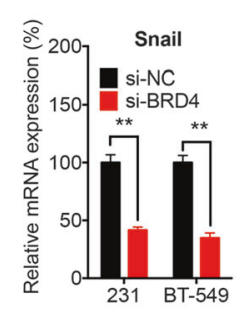

B

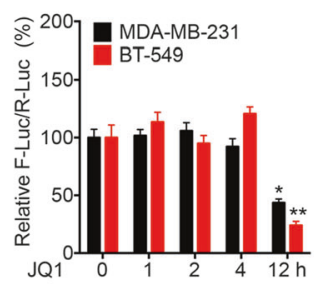

C

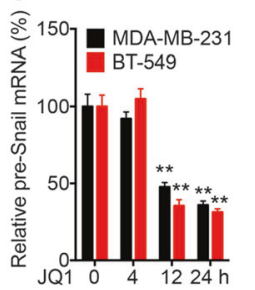

D

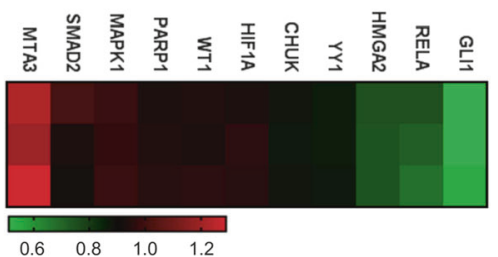

E

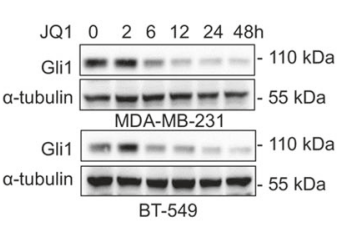

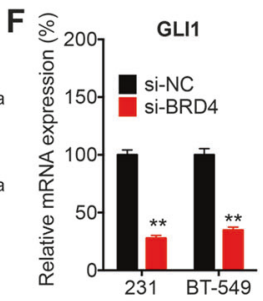

G

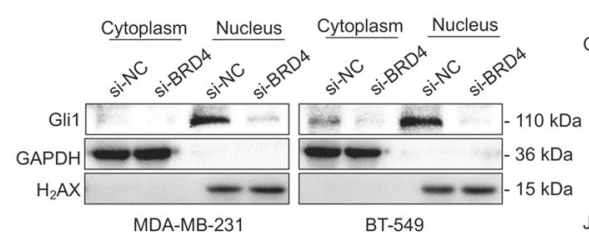

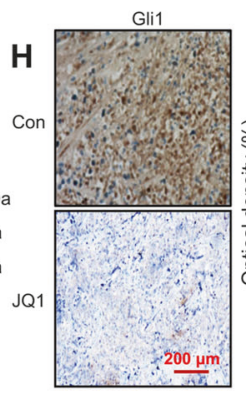

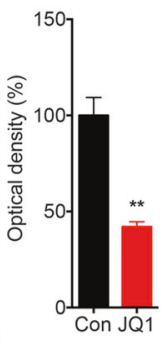

I
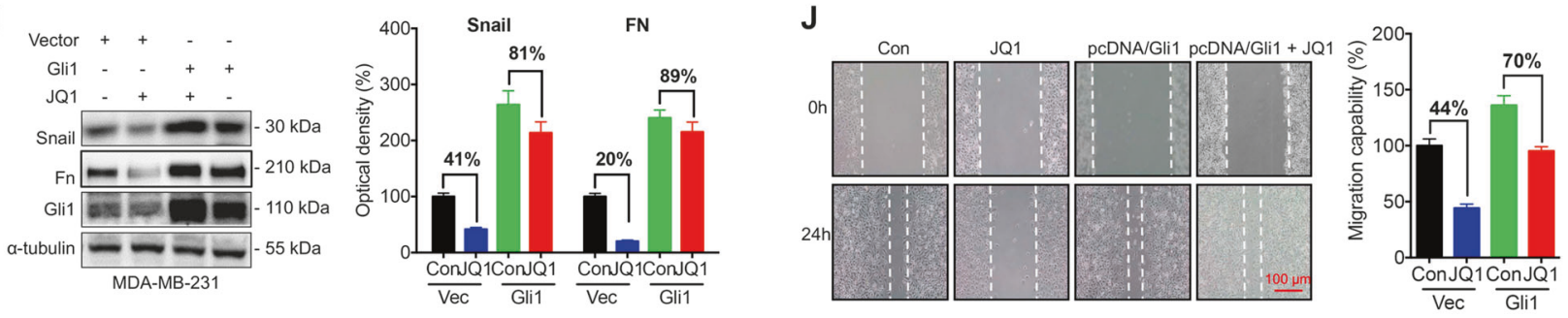

$\mathrm{K}$

$\begin{array}{cccc}\text { SITE } & \text { POSITION } & \text { HOMOLOGY } & \text { SEQUENCE } \\ \text { a } & -1588 \sim-1580 & 7 / 9(78 \%) & \text { GGGGTGATC } \\ \text { b } & -1374 \sim-1366 & 7 / 9(78 \%) & \text { TTGGTGGTG } \\ \text { c } & -1212 \sim-1204 & 7 / 9(78 \%) & \text { GCCCACCCC } \\ \text { d } & -597 \sim-589 & 7 / 9(78 \%) & \text { CGGGTGCTC } \\ \text { e } & -341 \sim-333 & 7 / 9(78 \%) & \text { GACCACCGG } \\ \text { f } & -44 \sim-36 & 7 / 9(78 \%) & \text { CACCACCCC }\end{array}$

Gli consensus sequence: GACCACCCA

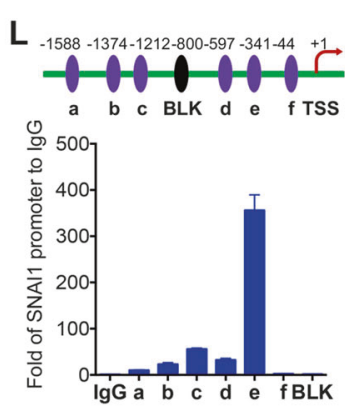

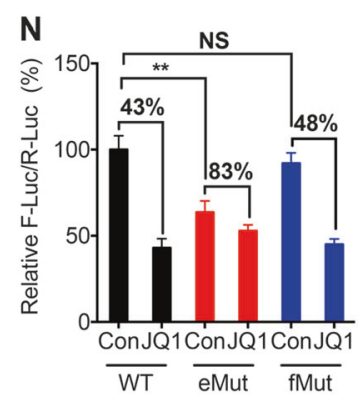

Fig. 5 BRD4 transcriptionally regulates Snail via Gli1. Cells were transfected with si-NC or si-BRD4 for $24 \mathrm{~h}$, the mRNA expression of Snail was checked by qRT-PCR; a Cells were transfected with pGL3Basic-Snail-luc reporter and pRL-TK plasmid for $24 \mathrm{~h}$ and then treated with JQ1 $(1 \mu \mathrm{M})$ for increasing time periods. Results were expressed as the ratio between the activity of the reporter plasmid and pRL-TK; b Cells were treated with JQ1 $(1 \mu \mathrm{M})$ for increasing time periods. PremRNA of Snail was checked by qRT-PCR; c MDA-MB-231 cells were treated with JQ1 $(1 \mu \mathrm{M})$ for $4 \mathrm{~h}$. The expression of transcription factors regulating Snail transcription was checked by qRT-PCR; d MDA-MB-231 cells were treated with JQ1 $(1 \mu \mathrm{M})$ for increasing time periods. Protein of Gli1 was checked by western blot analysis; e Cells were transfected with siNC or si-BRD4 for $24 \mathrm{~h}$. The mRNA expression of Gli1 was checked by qRT-PCR; f Cells were transfected with siNC or si-BRD4 for $24 \mathrm{~h}$. The Gli1 in subcellular fractions was checked by western blot analysis; $\mathbf{g}$ The expression of Gli1 in paraffinembedded sections obtained from xenografts was measured by IHC (left) and quantitatively analyzed (right); $\mathrm{h}$ The expression of Gli1 in paraffin-embedded sections obtained from xenografts was measured by IHC (left) and quantitatively analyzed (right); i, j MDA-MB-231 cells were transfected with pcDNA (vector) or pcDNA/Gli1 for $24 \mathrm{~h}$ and then treated with or without JQ1 $(1 \mu \mathrm{M})$ for $24 \mathrm{~h}$. i Protein expression was checked by western blot analysis. $\mathbf{j}$ Wound healing was recorded (left) and quantitatively analyzed (right); k Position in relation to the transcription start site and sequence homology to the Gli1 consensus binding sequence for putative Gli-binding sites in the human SNAII promoter. Red bases represent those different from the Gli-binding consensus sequence; I Binding between Gli1 transcriptional factor and the promoter of SNAII at the potential binding site "a" to "f" or negative site "BLK" was checked by ChIP-PCR; m Schematic representation of the mutated promoter in pGL3-BasicSnail-luc reporter to investigate the role of Gli1 in Snail expression; n MDA-MB-231 cells were transfected with pGL3-Snail-WT-Luc, pGL3-Snail-eMut-Luc, pGL3-Snail-fMut-Luc, and pRL-TK plasmid for $24 \mathrm{~h}$ and then treated with or without JQ1 $(1 \mu \mathrm{M})$ for $12 \mathrm{~h}$. Results were expressed as the ratio between the activity of the reporter plasmid and $\mathrm{pRL}-\mathrm{TK}$. Data are expressed as mean $\pm \mathrm{SD}$ and similar results were obtained from three independent experiments 
A

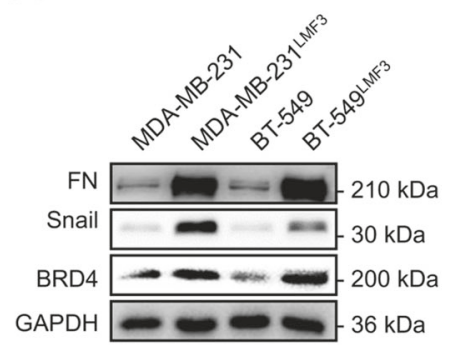

B

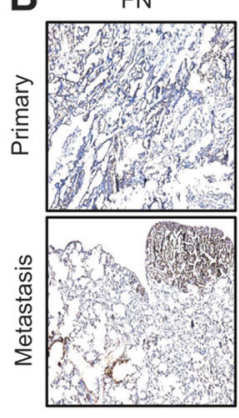

Snail

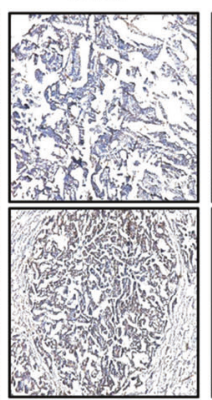

BRD4

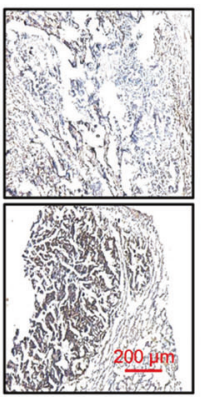

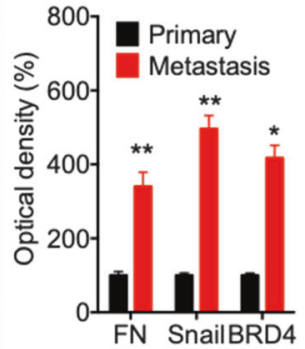

C

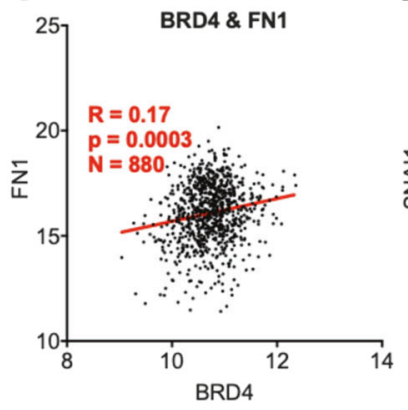

D

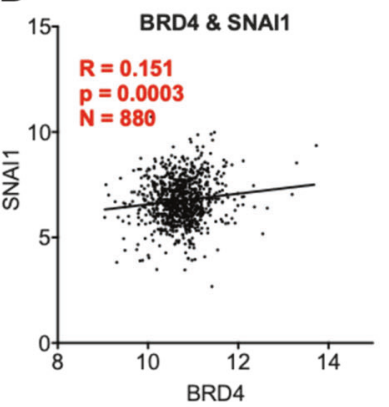

E

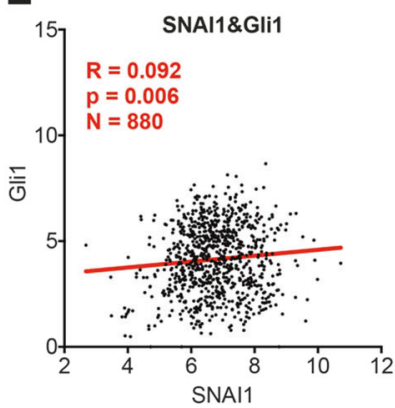

$\mathbf{F}$

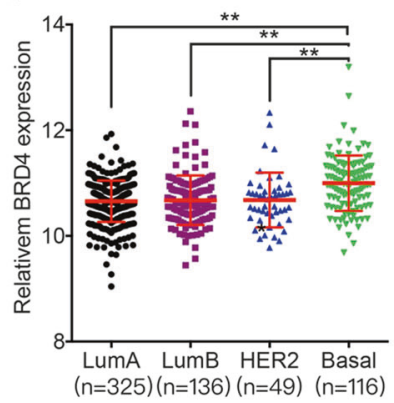

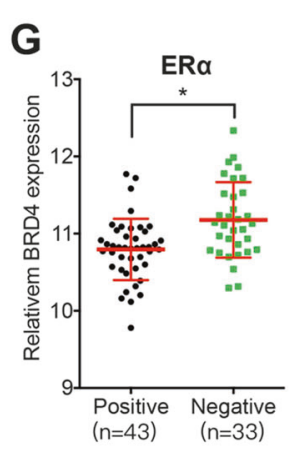

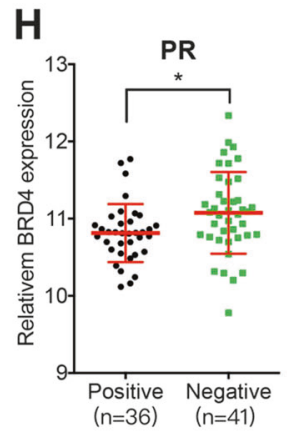

I.

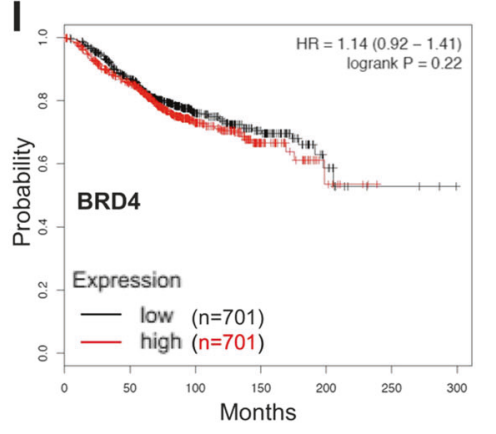

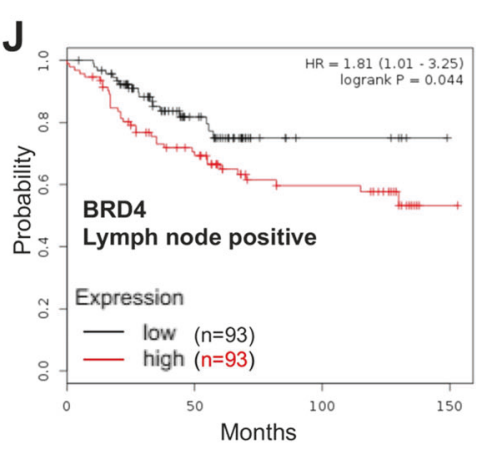

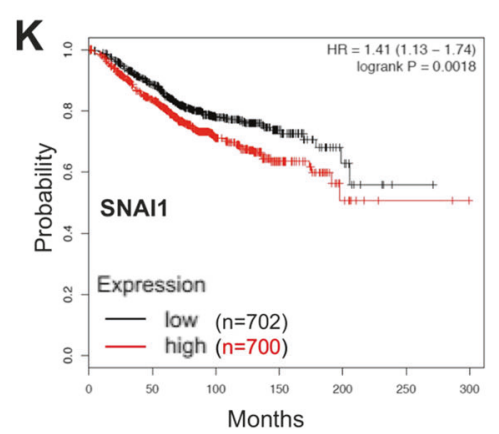

Fig. 6 BRD4/Snail axis on the progression of breast cancer. a Expression of FN, Snail, and BRD4 in MDA-MB-231 ${ }^{\text {LMF3 }}$ and BT-549 LMF3 cells and their corresponding parental cells were checked by western blot analysis; b The expression of FN, Snail, and BRD4 in primary tumors and metastasized tumors in lungs isolated from MMTV-PyMT mice was measured by IHC (left) and quantitatively analyzed (right); c-e Pearson correlation between BRD4 and FN1 (c), BRD4 and SNAI1 (d), and SNAI1 and Gli1 (e) in 880 breast cancer tissues from the TCGA database; $\mathbf{f}$ Relative mRNA expression of BRD4 in breast cancer subtypes including luminal A, Luminal B, HER2, and basal based on data available from the TCGA database; $g$ Relative mRNA expression of BRD4 in ER-positive and -negative breast cancer subtypes based on data available from the TCGA database; h Relative mRNA

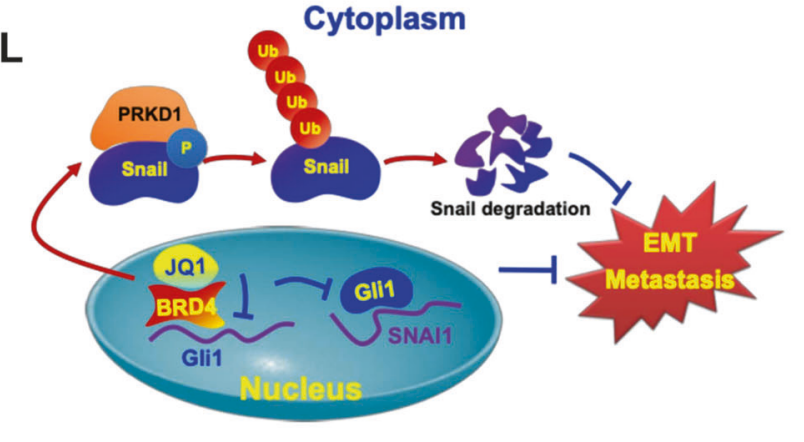

expression of BRD4 in PR-positive or -negative breast cancer subtypes based on data available from the TCGA database; i Overall survival (OS) in patients with high $(n=701)$ vs. low $(n=701)$ levels of BRD4 (202102_at) in BC patients plotted by the Kaplan-Meier method; j Overall survival (OS) in patients with high $(n=701)$ vs. low $(n=701)$ levels of BRD4 (202102_at) in lymph node-positive BC patients plotted by the Kaplan-Meier method; $\mathbf{k}$ Overall survival (OS) in patients with high $(n=702)$ vs. low $(n=700)$ levels of SNAI1 in BC patients plotted by the Kaplan-Meier method. I Proposed model to illustrate the mechanisms of targeted inhibition of BRD4-suppressed dissemination of BC cells via transcriptional and post-translational downregulation of Snail. Data were presented as means \pm SD from three independent experiments. $* p<0.05, * * p<0.01$ compared with control 
such as luminal A, B and HER2+BCs (Fig. 6f). In addition, expression of BRD4 in ERo-negative (Fig. $6 \mathrm{~g}$ ) and PRnegative (Fig. 6h) BCs were significantly $(p<0.05)$ greater than that in their positive BCs, respectively. Consistently, expression of SNAI1 in basal and HER2+BC was significantly $(p<0.01)$ greater than that in luminal A and B BCs (Fig. S6D). While expression of SNAI1 in ER $\alpha-$ (Fig. S6E) and PR- (Fig. S6F) BCs was significantly $(p<$ $0.05)$ greater than that in positive BCs.

Using the online bioinformatics tool Kaplan-Meier plotter [33], we found that BC patients with increased expression of BRD4 had no significant difference of overall survival (OS) with the $p$ value of 0.22 (Fig. 6i). However, in lymph node-positive BC patients, increased expression of BRD4 showed significantly reduced OS (Fig. 6j). Further, BC patients with increased expression of Snail showed significantly $(p=0.0018)$ reduced OS (Fig. 6k). Together, these results suggested that BRD4/Snail axis could trigger the progression of $\mathrm{BC}$.

\section{Discussion}

In this study, we examined the biological effects of targeted inhibition of BRD4 on the development of BC. We found that siRNA for BRD4 or a specific inhibitor of the BET family proteins (JQ1) suppresses the migration and EMT of BC cells via both transcriptional and post-translational downregulation of Snail (Fig. 61). Mechanistically, targeting BRD4 increases the expression of PRKD1 and then triggers the ubiquitination and degradation of Snail. Further, inhibition of BRD4 suppressed the transcription of Gli1, which binds the GACCACCGG sequence in the SNAII promoter to increase its transcription. Animal and clinical studies confirmed that BRD4/Snail pathway is critical for BC progression.

We found that targeted inhibition of BRD4 can suppress the growth and progression of $\mathrm{BC}$ in vitro and in vivo. The inhibitors of BET are currently in different phases of preclinical and clinical trials for treatment of multiple tumor types [5], including NUT midline carcinoma [34], multiple myeloma [35], and AML [36]. BET proteins are exciting targets for $\mathrm{BC}$ treatment to suppress cell growth and induce apoptosis [9, 37, 38]. Recently, BRD4 was shown to also regulate TNBC migration and invasion via regulating Jagged1/Notch1 signaling [10]. Disrupting the interaction of BRD4 with diacetylated Twist, another EMT-TF, can suppress invasion and tumorigenicity of basal-like BC cells [11]. We found that targeted inhibition of BRD4 suppresses the migration, invasion, and EMT of BC cells via downregulation of Snail, which confers tumor cells with cancer stem cell-like traits and promotes drug resistance, tumor recurrence, and metastasis [39].
We found that BRD4 regulates protein stability and transcription of Snail in BC cells. JQ1 can rapidly decrease Snail via increasing its ubiquitination in $\mathrm{BC}$ cells. Intriguingly, BRD4 interacts with Snail in BC cells; however, JQ1 had no effect on this interaction. Further, JQ1 had no effect on Snail acetylation in BC cells. The molecular nature of Snail-BRD4 interaction and whether other post-translational modification of Snail, besides the reported K187 acetylation [23], is involved in BRD4 association remain to be further investigated. By screening the factors involved in Snail stability, we found that targeted inhibition of BRD4 increases PRKD1, which can phosphorylate Sanil at Ser11 and trigger its degradation by E3 ligase FBXO11 [26]. We did not investigate the mechanisms responsible for JQ1induced upregulation of PRKD1. Multiple signaling pathways, such as $\beta$-catenin, NF-kB, MYC/MAX, androgen receptor, and $\mathrm{Sp} 1$, can regulate the transcription of PRKD1 [40]. BRD4 may intact with these signaling components to regulate the expression of PRKD1, which remains to be further investigated. Gli1, a key mediator of the Hedgehog (Hh) pathway for cancer progression [41], is responsible for BRD4-regulated transcription of Snail in BC cells. BRD4 can directly occupy GLII and GLI2 promoters, with a substantially decreased engagement at these sites after JQ1 treatment [42]. We further found that Gli1 can bind to the GACCACCGG sequence in the SNAII promoter and directly increase SNAI1 transcription. This finding contributes to our understanding of the molecular basis underpinning EMT regulation and cancer metastasis mediated by the Gli1 signaling pathway [29].

Increased expression of BRD4 and Snail has been observed in BC, particularly for more aggressive subtypes such as lung-metastasized BC, ER-, and PR- BCs. Consistently, BRD4 is a potential therapeutic target for triple-negative $[9,43]$ and metastasized [10] BC in recent studies. The expression of BRD4 correlates with the expression of ECM genes that constitute prominent components of metastasis-predictive gene expression signatures [44-47]. Similarly, the expression of BRD4 correlates with the expression of FN and MMPs in our present study. Our data, together with the published results, further highlight that BRD4 may be a potential target for $\mathrm{BC}$ therapy.

In summary, we showed that inhibition of BRD4 suppresses the dissemination and progression of BC via regulation of Snail. Our results provide strong interconnection between BRD4 and Snail to control EMT and $\mathrm{BC}$ cell migration and invasion, reinforcing the view that targeted inhibition of BRD4 is a promising therapeutic approach for aggressive and metastasized $\mathrm{BC}$ patients. 


\section{Materials and methods}

\section{Patient-derived xenograft preparation and JQ1 treatment}

All animal experiments were conducted according to the guidelines of the Animal Care and Use Committee of Sun Yat-sen University. Animals were maintained in accordance to the guidelines of the American Association of Laboratory Animal Care. A patient sample (48 years old) diagnosed with stage IV (metastatic) triple-negative breast cancer (TNBC) was acquired from the First Affiliated Hospital of Sun Yat-Sen University. Surgical materials were collected, cut into pieces and implanted in Non-obese diabetic (NOD)-SCID mice with $6 \mathrm{~h}$ of resection to generate F1 tumors. The PDX model was deemed viable if F1 tumors demonstrated progressive growth. When F1 tumor grew to about $400 \mathrm{~mm}^{3}$, the xenograft was harvested, homogenized, and transplanted into the subcutaneous tissue of the NOD-SCID mice $(n=12)$. When the tumor was visible, mice were randomized into two groups of five mice with similar average xenograft tumor volumes and assigned to a receive treatment with JQ1 $(50 \mathrm{mg} / \mathrm{kg}$ in $10 \%$ (2-hydroxypropyl)- $\beta$-cyclodextrin, b.i.d.) or vehicle only $(n=5$ xenografts per arm) every day for 20 days. Tumor size was measured every 2 days in two dimensions using a caliper. Tumor volume was calculated using the formula $V=1 / 2 \times$ larger diameter $\times(\text { smaller diameter })^{2}$. At the time of killing, tumors were weighed and tumor portions were snap-frozen and stored in liquid nitrogen or fixed in $10 \%$ buffered formalin for routine histopathologic processing.

\section{Establishment of breast cancer lung metastasis (BCLM) cell line models}

The establishment of breast cancer lung metastasis cell line models was conducted according to the previous study with slight modifications as summarized in Fig. S2 [21]. Briefly, to generate lung metastasis F1 (LMF1) cells, female nude mice (4 weeks old) were injected with $2 \times 10^{5}$ viable MDA-MB-231 or BT-549 cells into the lateral tail vein in a volume of $0.1 \mathrm{ml}$. Endpoint assays were conducted at 10 weeks post injection unless significant morbidity required that the mouse should be euthanized earlier. Then breast cancer cells metastasized to the lung were isolated, primarily cultured with medium, and named as MDA-MB-231 ${ }^{\mathrm{LMF} 1}$ or BT-549 ${ }^{\mathrm{LMF} 1}$, respectively. LMF2 and LMF3 cells were similarly generated from their corresponding parental cells. The LMF3 cells were used for further analysis and compared to the characteristics of F0 (parental) cells.

\section{Snail promoter activity assay}

Promoter activity of Snail in cells treated with or without JQ1 was measured according to our previously described protocol [48]. Briefly, cells were transfected with pGL3Snail-WT-Luc, pGL3-Snail-eMut-Luc, or pGL3-SnailfMut-Luc containing the $-1500 /+59$ sequence of the Snail promoter. Transfection efficiency was normalized by co-transfection with pRL-TK. After 24-h incubation, cells were analyzed with the Dual-Glo Luciferase Assay system (Promega). Renilla Luciferase (R-luc) was used to normalize firefly luciferase (F-luc) activity to evaluate the reporter translation efficiency.

\section{Database (DB) analysis}

The expression of BRD4 and Snail in BC and other cancers were analyzed by using data obtained from the Oncomine Database (www.oncomine.org). The sample information and expression data are available in the Gene Expression Omnibus (GEO) database [Accession nos. GSE9014 (Finak Breast) and EGAS00000000083 (Cutis Breast) at www. ncbi.nlm.nih.gov/geo].

The expression profiles of Snail, BRD4, PRKD1, and Gli1 among the subtypes of BC patients were downloaded from LinkedOmics (http://www.linkedomics.org), which is a publicly available portal that includes multi-omics data from all of 32 cancer types from The Cancer Genome Atlas (TCGA) project. The LinkedOmics web allowed flexible exploration of associations between a molecular or clinical attribute of interest and all other attributes, providing the opportunity to analyze and visualize associations between billions of attribute pairs for each cancer cohort [32].

\section{Statistical analysis}

Results were expressed as mean \pm standard deviation (SD) of three independent experiments unless otherwise specified. Data were analyzed by two-tailed unpaired Student's ttest between any two groups. These analyses were performed using GraphPad Prism Software Version 5.0 (GraphPad Software Inc., La Jolla, CA). Image J software was used to analyze the data from wound-healing, transwell and Western blot analyses. $P$-value of $<0.05$ was considered statistically significant.

Acknowledgements We thank the help of Dr. Shuibin Lin and Dr. Ge Zhang at Sun Yat-sen University for suggestions and discussions.

Funding This research was supported by the National Natural Science Foundation of China (Grant Nos. 81673454, 81672608, and 81472470), the National Natural Science Foundation Key Project of China (Grant no. 81430041), the Guangdong Natural Science Funds for Distinguished Young Scholar (No. 2014A030306025), the 
Guangdong Provincial Key Laboratory of Construction Foundation (No. 2017B030314030), the Guangdong Province Key Laboratory of Malignant Tumor Epigenetics and Gene Regulation (2017B030314026), and the Fundamental Research Funds for the Central Universities (Sun Yat-sen University) (No.16ykpy09). CM Chiang's research was supported by US National Institutes of Health (CA103867), Cancer Prevention Research Institute of Texas (RP180349 and RP190077), and the Welch Foundation (I-1805).

Author contributions Conception and design: HS Wang, ZJ Chen, LL Lu, H Shan, and L Tian; Acquisition of data: LL Lu, XY Lin, WG Li, $\mathrm{YM} \mathrm{Wu}$, and PP An; Analysis and interpretation of data: HS Wang, CM Chiang, ZJ Chen, J Du, and Q Su; Writing, review, and/or revision of the manuscript: HS Wang, CM Chiang, LL Lu, and H Shan.

\section{Compliance with ethical standards}

Conflict of interest The authors declare that they have no conflict of interest.

Informed consent The authors confirmed that we have obtained written consent from the patient to publish the manuscript.

Publisher's note: Springer Nature remains neutral with regard to jurisdictional claims in published maps and institutional affiliations.

\section{References}

1. Wu SY, Chiang CM. The double bromodomain-containing chromatin adaptor Brd4 and transcriptional regulation. J Biol Chem. 2007;282:13141-5.

2. Belkina AC, Denis GV. BET domain co-regulators in obesity, inflammation and cancer. Nat Rev Cancer. 2012;12:465-77.

3. Chiang CM. Brd4 engagement from chromatin targeting to transcriptional regulation: selective contact with acetylated histone $\mathrm{H} 3$ and H4. F1000 Biol Rep. 2009;1:98.

4. Kanno T, Kanno Y, LeRoy G, Campos E, Sun HW, Brooks SR, et al. BRD4 assists elongation of both coding and enhancer RNAs by interacting with acetylated histones. Nat Struct Mol Biol. 2014;21:1047-57.

5. Filippakopoulos P, Knapp S. Targeting bromodomains: epigenetic readers of lysine acetylation. Nat Rev Drug Discov. 2014;13:337-56.

6. Zuber J, Shi JW, Wang E, Rappaport AR, Herrmann H, Sison EA, et al. RNAi screen identifies Brd4 as a therapeutic target in acute myeloid leukaemia. Nature. 2011;478:524-U124.

7. Chapuy B, McKeown MR, Lin CY, Monti S, Roemer MG, Qi J, et al. Discovery and characterization of super-enhancer-associated dependencies in diffuse large B cell lymphoma. Cancer Cell. 2013;24:777-90.

8. Asangani IA, Dommeti VL, Wang X, Malik R, Cieslik M, Yang $\mathrm{R}$, et al. Therapeutic targeting of BET bromodomain proteins in castration-resistant prostate cancer. Nature. 2014;510:278-82.

9. Shu S, Lin CY, He HH, Witwicki RM, Tabassum DP, Roberts $\mathrm{JM}$, et al. Response and resistance to BET bromodomain inhibitors in triple-negative breast cancer. Nature. 2016;529:413-7.

10. Andrieu G, Tran AH, Strissel KJ, Denis GV. BRD4 regulates breast cancer dissemination through Jagged1/Notch1 signaling. Cancer Res. 2016;76:6555-67.

11. Shi J, Wang YF, Zeng L, Wu YD, Deng J, Zhang Q, et al. Disrupting the interaction of BRD4 with diacetylated Twist suppresses tumorigenesis in basal-like breast cancer. Cancer Cell. 2014;25:210-25.
12. Thiery JP, Acloque H, Huang RYJ, Nieto MA. Epithelialmesenchymal transitions in development and disease. Cell. 2009;139:871-90.

13. Puisieux A, Brabletz T, Caramel J. Oncogenic roles of EMTinducing transcription factors. Nat Cell Biol. 2014;16:488-94.

14. Lin YW, Wu YD, Li JL, Dong CF, Ye XF, Chi YI, et al. The SNAG domain of Snail1 functions as a molecular hook for recruiting lysinespecific demethylase 1. EMBO J. 2010;29:1803-16.

15. Dong C, Wu Y, Wang Y, Wang C, Kang T, Rychahou PG, et al. Interaction with Suv39H1 is critical for Snail-mediated E-cadherin repression in breast cancer. Oncogene. 2013;32:1351-62.

16. Zhou Y, Lu L, Jiang G, Chen Z, Li J, An P, et al. Targeting CDK7 increases the stability of Snail to promote the dissemination of colorectal cancer. Cell Death Differ. 2018. https://doi.org/10. 1038/s41418-018-0222-4

17. Filippakopoulos P, Qi J, Picaud S, Shen Y, Smith WB, Fedorov $\mathrm{O}$, et al. Selective inhibition of BET bromodomains. Nature. 2010;468:1067-73.

18. Ba MC, Long H, Yan ZF, Wang S, Wu YB, Tu YN, et al. BRD4 promotes gastric cancer progression through the transcriptional and epigenetic regulation of c-MYC. J Cell Biochem. 2018;119:973-82.

19. Puissant A, Frumm SM, Alexe G, Bassii CF, Qi J, Chanthery YH, et al. Targeting MYCN in neuroblastoma by BET bromodomain inhibition. Cancer Res. 2013;73:308-23.

20. Delmore JE, Issa GC, Lemieux ME, Rahl PB, Shi JW, Jacobs $\mathrm{HM}$, et al. BET bromodomain inhibition as a therapeutic strategy to target c-Myc. Cell. 2011;146:903-16.

21. Minn AJ, Gupta GP, Siegel PM, Bos PD, Shu WP, Giri DD, et al. Genes that mediate breast cancer metastasis to lung. Nature. 2005;436:518-24.

22. Davis FM, Stewart TA, Thompson EW, Monteith GR. Targeting EMT in cancer: opportunities for pharmacological intervention. Trends Pharmacol Sci. 2014;35:479-88.

23. Chang R, Zhang Y, Zhang P, Zhou Q. Snail acetylation by histone acetyltransferase p300 in lung cancer. Thorac Cancer. 2017;8:131-7.

24. Zhou BP, Deng J, Xia W, Xu J, Li YM, Gunduz M, et al. Dual regulation of Snail by GSK-3beta-mediated phosphorylation in control of epithelial-mesenchymal transition. Nat Cell Biol. 2004;6:931-40.

25. Wu Y, Deng J, Rychahou PG, Qiu S, Evers BM, Zhou BP. Stabilization of snail by NF-kappaB is required for inflammationinduced cell migration and invasion. Cancer Cell. 2009;15:416-28.

26. Du C, Zhang CY, Hassan S, Biswas MHU, Balaji KC. Protein kinase D1 suppresses epithelial-to-mesenchymal transition through phosphorylation of Snail. Cancer Res. 2010;70:7810-9.

27. Gustafsson MK, Pan H, Pinney DF, Liu YL, Lewandowski A, Epstein DJ, et al. Myf5 is a direct target of long-range Shh signaling and Gli regulation for muscle specification. Gene Dev. 2002;16:114-26.

28. Singh RR, Kunkalla K, Qu C, Schlette E, Neelapu SS, Samaniego $\mathrm{F}$, et al. ABCG2 is a direct transcriptional target of hedgehog signaling and involved in stroma-induced drug tolerance in diffuse large B-cell lymphoma. Oncogene. 2011;30:4874-86.

29. Li X, Deng W, Nail CD, Bailey SK, Kraus MH, Ruppert JM, et al. Snail induction is an early response to Gli1 that determines the efficiency of epithelial transformation. Oncogene. 2006;25:609-21.

30. Kong Y, Peng Y, Liu Y, Xin H, Zhan X, Tan W. Twist1 and Snail link Hedgehog signaling to tumor-initiating cell-like properties and acquired chemoresistance independently of $\mathrm{ABC}$ transporters. Stem Cells. 2015;33:1063-74.

31. Kinzler KW, Vogelstein B. The GLI gene encodes a nuclear protein which binds specific sequences in the human genome. Mol Cell Biol. 1990;10:634-42. 
32. Vasaikar SV, Straub P, Wang J, Zhang B. LinkedOmics: analyzing multi-omics data within and across 32 cancer types. Nucleic Acids Res. 2018;46(D1):D956-D963.

33. Szasz AM, Lanczky A, Nagy A, Forster S, Hark K, Green JE, et al. Cross-validation of survival associated biomarkers in gastric cancer using transcriptomic data of 1,065 patients. Oncotarget. 2016;7:49322-33.

34. Stathis A, Zucca E, Bekradda M, Gomez-Roca C, Delord JP, de La Motte Rouge T, et al. Clinical response of carcinomas harboring the BRD4-NUT oncoprotein to the targeted bromodomain inhibitor OTX015/MK-8628. Cancer Discov. 2016;6:492-500.

35. Amorim S, Stathis A, Gleeson M, Iyengar S, Magarotto V, Leleu $\mathrm{X}$, et al. Bromodomain inhibitor OTX015 in patients with lymphoma or multiple myeloma: a dose-escalation, open-label, pharmacokinetic, phase 1 study. Lancet Haematol. 2016;3: e196-204.

36. Berthon C, Raffoux E, Thomas X, Vey N, Gomez-Roca C, Yee K, et al. Bromodomain inhibitor OTX015 in patients with acute leukaemia: a dose-escalation, phase 1 study. Lancet Haematol. 2016;3:e186-195.

37. Yang L, Zhang Y, Shan W, Hu Z, Yuan J, Pi J, et al. Repression of BET activity sensitizes homologous recombination-proficient cancers to PARP inhibition. Sci Transl Med. 2017;9:eaal1645.

38. Lai X, Stiff A, Duggan M, Wesolowski R, Carson WE 3rd, Friedman A. Modeling combination therapy for breast cancer with BET and immune checkpoint inhibitors. Proc Natl Acad Sci USA. 2018;115:5534-9.

39. Wang Y, Shi J, Chai K, Ying X, Zhou BP. The role of Snail in EMT and tumorigenesis. Curr Cancer Drug Targets. 2013;13: 963-72.

40. Nickkholgh B, Sittadjody S, Rothberg MB, Fang XL, Li KZ, Chou JW, et al. Beta-catenin represses protein kinase D1 gene expression by non-canonical pathway through MYC/MAX transcription complex in prostate cancer. Oncotarget. 2017;8: 78811-24.

41. Amakye D, Jagani Z, Dorsch M. Unraveling the therapeutic potential of the Hedgehog pathway in cancer. Nat Med. 2013;19: 1410-22.

42. Tang YJ, Gholamin S, Schubert S, Willardson MI, Lee A, Bandopadhayay $\mathrm{P}$, et al. Epigenetic targeting of Hedgehog pathway transcriptional output through BET bromodomain inhibition. Nat Med. 2014;20:732-40.

43. da Motta LL, Ledaki I, Purshouse K, Haider S, De Bastiani MA, Baban D, et al. The BET inhibitor JQ1 selectively impairs tumour response to hypoxia and downregulates CA9 and angiogenesis in triple negative breast cancer. Oncogene. 2017;36:122-32.

44. van 't Veer LJ, Dai H, van de Vijver MJ, He YD, Hart AA, Mao $\mathrm{M}$, et al. Gene expression profiling predicts clinical outcome of breast cancer. Nature. 2002;415:530-6.

45. van de Vijver MJ, He YD, van 't Veer LJ, Dai H, Hart AAM, Voskuil DW, et al. A gene-expression signature as a predictor of survival in breast cancer. New Engl J Med. 2002;347: 1999-2009.

46. Crawford NPS, Alsarraj J, Lukes L, Walker RC, Officewala JS, Yang $\mathrm{HH}$, et al. Bromodomain 4 activation predicts breast cancer survival. Proc Natl Acad Sci USA. 2008;105:6380-5.

47. Wu SY, Nin DS, Lee AY, Simanski S, Kodadek T, Chiang CM. BRD4 phosphorylation regulates HPV E2-mediated viral transcription, origin replication, and cellular MMP-9 expression. Cell Rep. 2016;16:1733-48.

48. Jiang GM, Wang HS, Zhang F, Zhang KS, Liu ZC, Fang R, et al. Histone deacetylase inhibitor induction of epithelial-mesenchymal transitions via up-regulation of Snail facilitates cancer progression. Biochim Biophys Acta. 2013;1833:663-71. 